\title{
A Case of a Young Asymptomatic Woman with Optic Disc Drusen and Vasculitis
}

\author{
Tryfon Rotsos Chrysanthos Symeonidis Mrinal Rana \\ James S. Deane \\ Department of Ophthalmology, Leicester Royal Infirmary, University Hospitals of \\ Leicester NHS Trust, Leicester, UK
}

\section{Key Words}

Optic disc drusen · Retinal periphlebitis - Optical coherence tomography

\begin{abstract}
Purpose: We present the case of a young woman with optic disc drusen and peripheral vasculitis.
\end{abstract}

Methods: Diagnosis was based on fundoscopic, optical coherence tomography as well as fluorescein angiography (FA) findings.

Results: An asymptomatic 34-year-old female patient with no systemic pathology was referred to our hospital from her optician for retinal findings. Fundoscopy revealed mild disc swelling that could be attributed to the presence of optic disc drusen in both eyes. There was fundoscopic evidence of periphlebitis in the periphery confirmed by FA findings.

Conclusions: In our case, the unique feature was the presence of optic disc drusen and retinal periphlebitis. The patient's disc configuration may have contributed to a predisposition for vasculitis in addition to vessel tortuosity.

\section{Introduction}

Small optic discs with abnormal vasculature have been shown to be predisposed to obstruction in axoplasmic flow. This may result in stasis, thus disrupting axonal metabolism. In these pathologic circumstances, calcium crystals are deposited into nerve cell mitochondria at first and then in the extracellular space. Constant calcification as well as increase in size of these deposits gives rise to optic disc drusen [1]. Retinal vasculitis is a pathological entity that may potentially lead to blindness. In its active stage, exudates are observed around retinal vessels thus resulting in cuffing or white sheathing of the affected vessels. Recurring vasculitic attacks eventually result in permanent vascular occlusion and 
tissue hypoxia, which in turn may cause neovascularization in the optic nerve head or elsewhere [2]. We present the case of a young asymptomatic woman with optic disc drusen and vasculitis.

\section{Case Report}

A 34-year-old female patient was referred to our hospital from her optician for retinal findings. The patient herself was asymptomatic with no systemic pathology. The patient was examined in the Medical Retina clinic. Baseline visual acuity was $6 / 5-2$ in the right eye and $6 / 5$ in the left eye. Anterior segment examination revealed no signs of active inflammation in both eyes. Intraocular pressures were $16 \mathrm{~mm}$ Hg in both eyes. Fundoscopy in the right eye revealed vessel tortuosity and dot and blot haemorrhages in the periphery and in the mid periphery. There was also mild disc swelling that could be attributed to the presence of optic disc drusen (fig. 1). From the left eye, less vessel tortuosity was noted. Optic disc drusen were also present in the left eye. No inflammatory activity (cells or vitreous haze), neovascularization or vitreous haemorrhage was observed in either eye.

Gonioscopy revealed no significant findings. Optical coherence tomography (OCT) revealed the presence of optic disc drusen and normal fovea in both eyes (fig. 2). Fluorescein angiography (FA) revealed marked leakage and venous sheathing from the peripheral and mid-peripheral veins in the right eye. Vascular remodeling and areas of ischaemia were evident. Less leakage was present from the infero-temporal vein branch (fig. 3). Findings in the left eye were much more subtle. The overall impression was consistent with peripheral vasculitis. Optic disc drusen presence was confirmed with optic nerve head ultrasonography (fig. 4).

No treatment was deemed necessary for this potentially longstanding vasculitis.

Chest X-ray was performed with no significant findings. Extensive blood tests were carried out, including potassium, sodium, urea, creatinine, albumin, alkaline phosphatase, gamma-glutamyl transferase levels, C-reactive protein, ESR, complete blood count, plasma viscosity and glomerular filtration rate. Thrombin, partial thromboplastin time, haemoglobin, bilirubin as well as anticardiolipin antibodies, haemoglobinopathies and quantiferon gold tube tuberculosis tests were also ordered. Serum ACE and VDRL tests were also carried out. All requested test results were normal. She also tested HLAB51 negative. We examined the patient 1 month later and there was no significant change in the patient's overall condition.

After 9 months visual acuity, fundoscopy, angiographic findings and OCT images were unaltered.

\section{Discussion}

Abnormal anatomic disc features such as small optic disc, tilted disc, and optic nerve drusen have been identified as discs at increased risk for several ophthalmic, neurological and vascular disorders [3]. In the relevant literature, optic disc drusen have been associated with small, crowded discs which may be predisposed to axonal flow obstruction due to smaller than average scleral foramina. The presence of optic disc drusen and chronic venous stasis at the optic nerve head may also result in vessel tortuosity varying with the degree of disc crowding. This optic nerve head configuration may render it susceptible to vascular diseases [4]. OCT has been recently shown to demonstrate and differentiate optic nerve drusen but is not yet considered a gold standard [5]. The presence of optic disc drusen may be confirmed with optic nerve head ultrasound or fundus autofluorescence [6].

In our case, venous stasis was observed in the right eye but not in the left eye. Periphlebitis in the mid-periphery was also observed in the right eye but it did not affect visual acuity in this eye. Signs of periphlebitis include gorged vessels, venous sheathing, 
localized ischaemia and haemorhage as well as vascular remodelling. Despite extensive laboratory investigations, no clear aetiology was identified for this periphlebitis as all potential inflammatory causes were excluded. Idiopathic periphlebitis represents $70-75 \%$ of the reported periphlebitis cases in the relevant literature. In rare occasions, optic disc drusen may be accompanied by vascular complications such as choroidal neovascularization. In this case, however, this possibly longstanding vasculitis did not require topical or systemic treatment.

The unique feature of this case is the presence of optic disc drusen and retinal periphlebitis. The patient's disc configuration may have contributed to a predisposition for vasculitis in addition to vessel tortuosity.

\section{Disclosure Statement}

The authors report no proprietary interest or financial support.

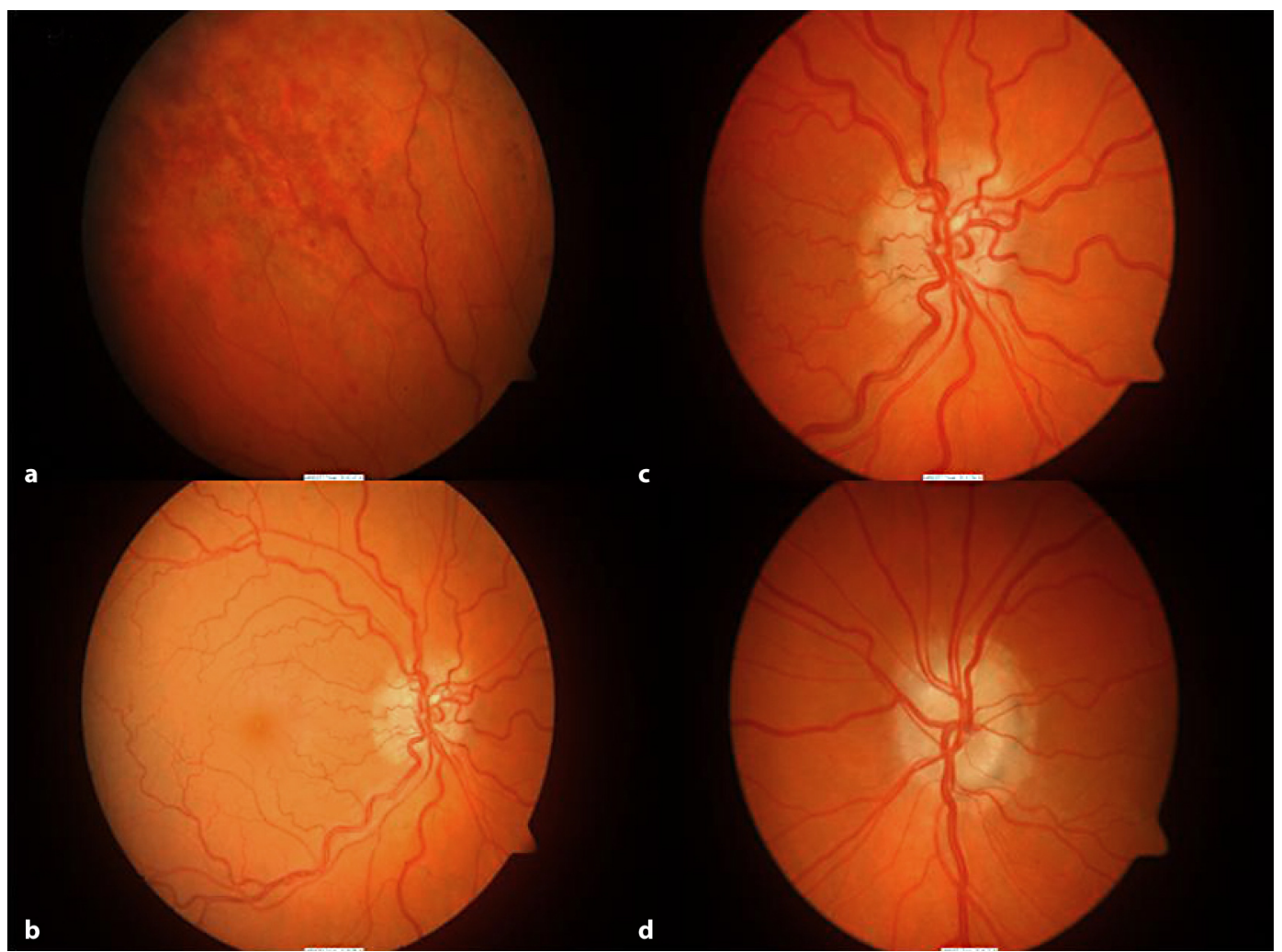

Fig. 1. Colour fundus photographs in our case. a Dot and blot hemorrhages in the periphery and in the mid periphery of the right eye were observed. b Right eye optic disc drusen. c Vessel tortuosity from the right eye. $\mathbf{d}$ Optic disc drusen in the left eye. 


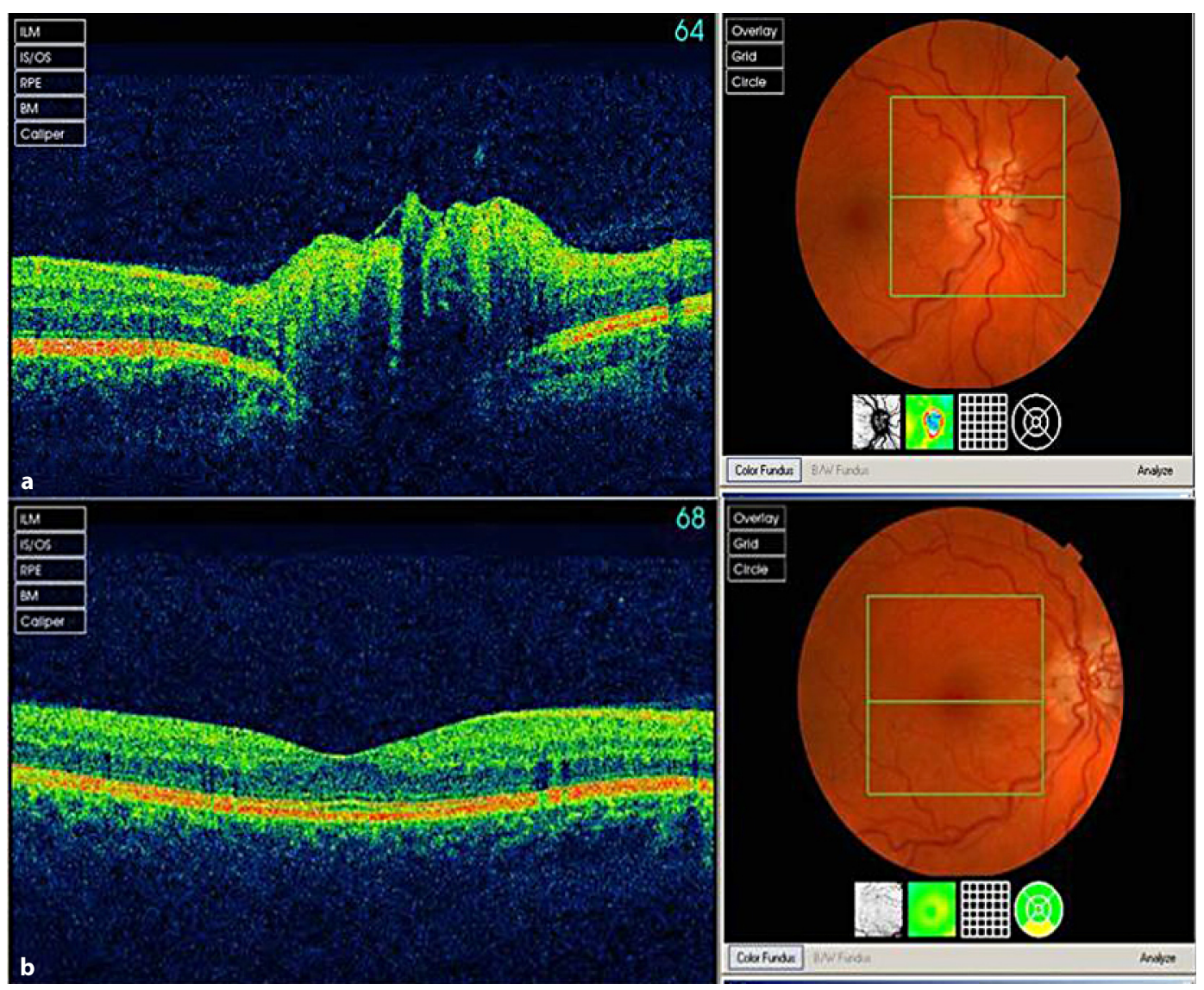

Fig. 2. OCT scans in our case. a OCT revealed optic disc drusen. b Fovea from the right eye was normal with no signs of subretinal or intraretinal fluid. 


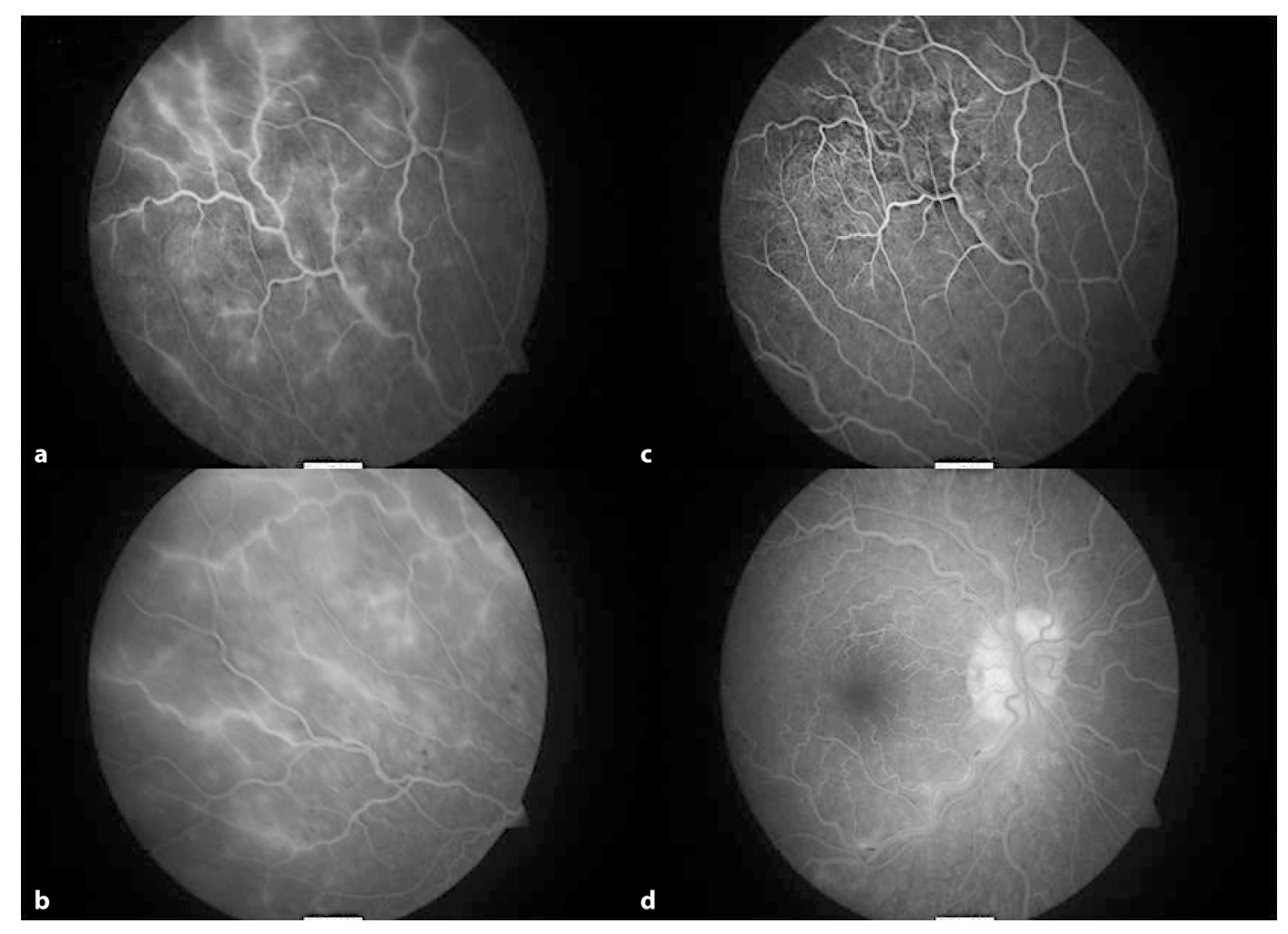

Fig. 3. FA photographs in our case. a Marked leakage and venous sheathing from the right eye was evident. b Vascular remodeling was revealed by FA. c Areas of ischaemia were revealed in the periphery in the right eye. $\mathbf{d}$ More subtle leakage was present from the infero-temporal vein branch. 


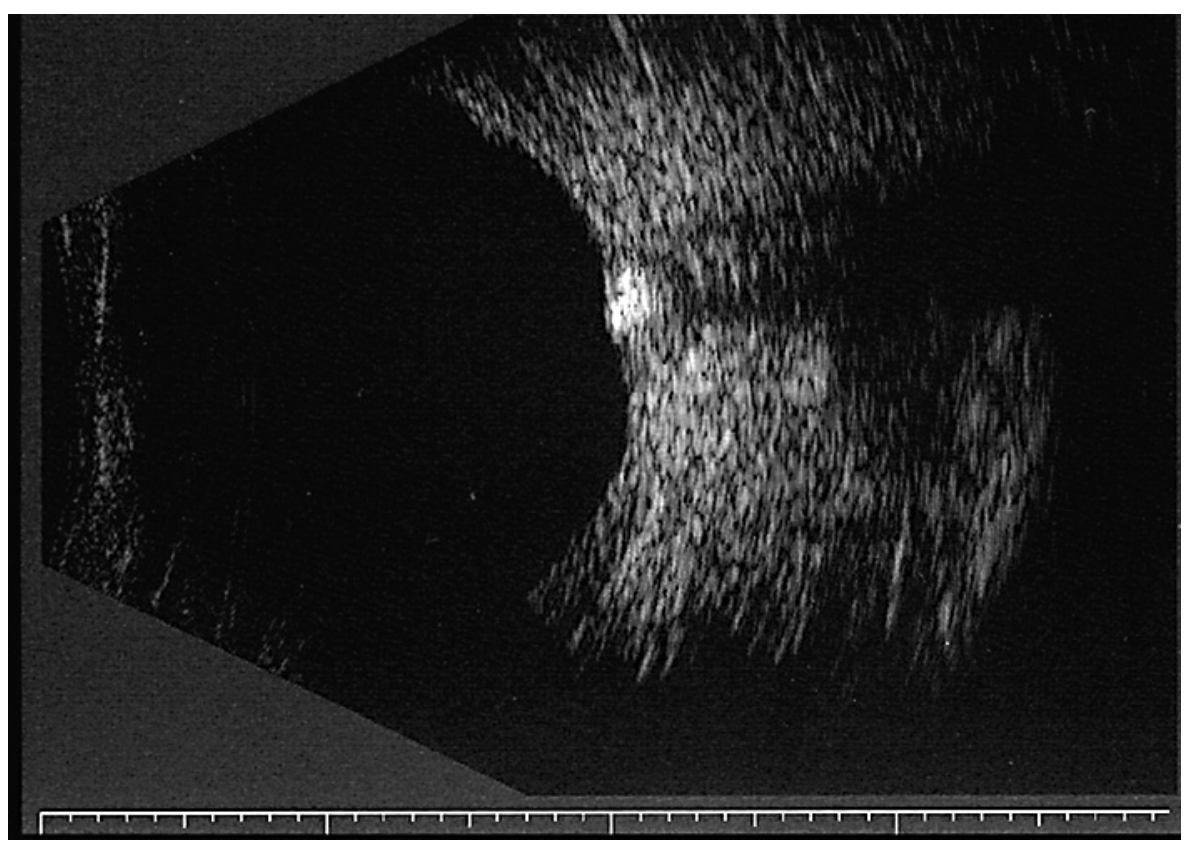

Fig. 4. Ultrasonography of the optic nerve head: optic disc drusen.

\section{References}

1 Lam BL, Morais CG Jr, Pasol J: Drusen of the optic disc. Curr Neurol Neurosci Rep 2008;8:404-408.

2 Wakakura M, Ishikawa S: Neuro-ophthalmic aspects of vascular disease. Curr Opin Ophthalmol 1994;5:18-22.

-3 Abu El-Asrar AM, Herbort CP, Tabbara KF: A clinical approach to the diagnosis of retinal vasculitis. Int Ophthalmol 2010;30:149-173.

4 Aumiller MS: Optic disc drusen: complications and management. Optometry 2007;78:10-16.

5 Patel NN, Shulman JP, Chin KJ, Finger PT: Optical coherence tomography/scanning laser ophthalmoscopy imaging of optic nerve head drusen. Ophthalmic Surg Lasers Imaging 2010;41:614-621.

-6 Friedman AH, Beckerman B, Gold DH, Walsh JB, Gartner S: Drusen of the optic disc. Surv Ophthalmol 1977;21:373-390. 